\title{
Vector Reconstruction and Mosaicing from Multiple Doppler Weather Radar Velocity Data
}

\author{
Pravas R. Mahapatra \\ Department of Aerospace Engineering \\ Indian Institute of Science \\ Bangalore - 560 012, India \\ Email: pravas@aero.iisc.ernet.in
}

\author{
Vishnu V. Makkapati \\ Honeywell Technology Solutions Lab \\ 151/1, Doraisanipalya, Bannerghatta Road \\ Bangalore - 560 076, India \\ Email: vishnu.makkapati@honeywell.com
}

\begin{abstract}
Radial velocity data from two or more Doppler weather radars are commonly combined to obtain the vector or true velocity of winds at a given location. This paper proposes a novel scheme for combining scalar and vector data from multiple radars. The number of overlapping radars at a point is automatically determined. The data combining algorithm (2-D, 3$D$, or least-squares) is automatically determined from the number of overlapping radars. The use of latitude-longitude grid results in a universal grid presentation. A variable (user-definable) boxaveraging method of vector representation is adopted and vector fields are depicted as fields of arrows. A novel method is adopted for handling boxes lying on boundaries of overlapping radar zones.
\end{abstract}

\section{INTRODUCTION}

Doppler weather radars (DWRs) such as the WSR-88D generate three raw moments of the hydrometeor distribution within each resolution cell. These correspond to reflectivity (indicating rainfall intensity), radial (Doppler) wind velocity (relative to the radar location), and spectrum width of the Doppler velocity spread. Mosaiced reflectivity data from a network of WSR-88Ds are also commonly available. However, the radial velocity data are not available from the archives in mosaiced form. Any combination of such data from multiple radars is left to be done by the user(s).

A common reason for combining radial velocity data from two or more DWRs is to obtain the vector or true velocity of winds at a given location. Performing this operation at points (or small area elements) over an extended region leads to mosaicing of velocity data similar to that of the reflectivity data. However, because of the vector nature of velocity data, their mosaicing is more complex than that of reflectivity. In this paper we present an algorithm for combining Doppler velocity data from multiple WSR-88D radars to generate a vector wind field extending over wide areas. The basic principles of obtaining the vector velocity have been known since the early days of DWR (e.g. [1], [2]) and have been implemented for local areas (e.g. [3], [4]). However, several practical problems are encountered in combining and mosaicing velocity data over large regions. Addressing these issues is necessary for a vector mosaicing program to be practically useful. The algorithm presented in this paper addresses the following issues:

- Variability of the number of radar coverage volumes overlapping at a given location. These may range from
0 (no radar coverage) or 1 (coverage by a single radar) to coverage by several radars. A single radar coverage yields no vector data, overlap of two radars yields a planar vector, a 3-radar overlap can produce a spatial (3-D) vector or redundant equations for constructing a 2-D vector, and simultaneous coverage of a point by 4 or more radars will yield redundant equations for 2- or 3-D vector reconstruction which may then be optimized.

- Variability of the size and orientation of the radar resolution volumes of multiple radars at a given location.

- The conical nature of the individual radar beam scanning surfaces for different elevation angles, and the nonoverlap of these conical surfaces from adjacent radars.

- Non-contiguous stacking of the scanning radar beams, i.e. presence of significant gaps between the scanning elevations of a radar, especially at the higher elevation angles.

- The curvature of the earth, which makes even the flat, zero-elevation scanning planes of adjacent radars to be non-overlapping, and requires additional formulation for transforming the coordinates from one radar to another, or to a common set of coordinates.

Recently, schemes have emerged for combining scalar reflectivity information from overlapping radars. The approach explained in [3] converts volume scan data of each individual radar from conical to cartesian coordinates and uses a weighted average scheme based on Cressman weighting function for obtaining a mosaic. US patent [5] uses a Look-up-table (LUT) for converting the radar data bins to the desired coordinate system and converts each of a plurality of radar data bins to a corresponding grid location or box in a mosaic image. Any resulting holes in the mosaic are filled with data from the closest data bin. US patent [6] avoids the drawbacks of mutual exclusion of the radar images so that a given point of the mosaic corresponds to the display on one and only one radar. However, these schemes use coarse cartesian grids, resulting in significant loss of spatial resolution while the conversion of coordinates from spherical to cartesian system is highly computation intensive.

The scheme proposed in [4] considers the radar measurements as spatially averaged volumes, instead of treating them 


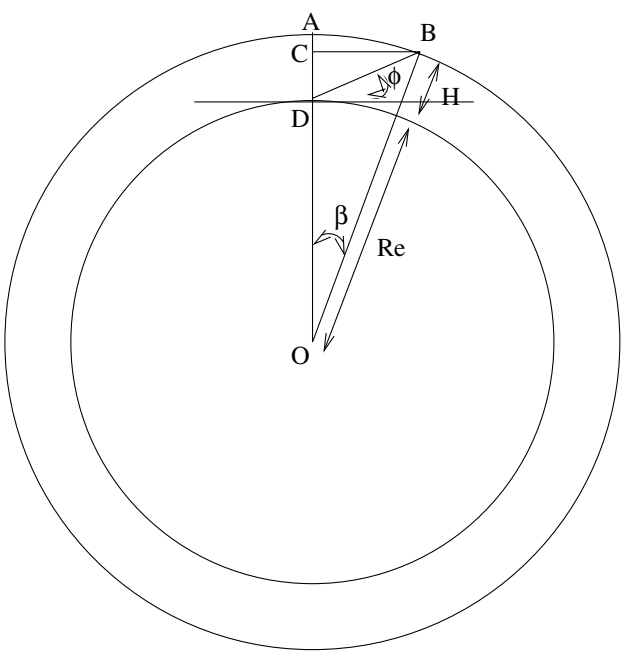

Fig. 1. Scheme for conversion of earth spherical coordinates to radar coordinates

as point values and uses a common volume model for combining data from overlapping radars. This scheme estimates the cartesian components of horizontal velocity using data from at least two spatially separated radars while the vertical velocity is calculated using the divergence in the horizontal plane and an equation of continuity. This scheme does not take into account the number of overlapping radars at a given grid point. However, an efficient solution can be provided, if the number of overlapping radars is considered.

\section{Constant Altitude Plan Position Indicator}

The present scheme represents the radar data in interpolative spherical-earth Constant-Altitude Plan Position Indicator (CAPPI) format for each radar before combining data from multiple radars. CAPPI is a horizontal slice taken through the radar volume scan data at constant altitude above the surface of the earth. CAPPI displays the weather conditions observed within a half-spherical observation area. The radar antenna transmits and receives pulses at different elevation angles and at different azimuth angles by both performing a rotating scan operation in the azimuth and by varying the elevation angle.

A conventional radar system suffers from a disadvantage in that it deteriorates the time resolution for observation due to the large time required in observing areas. The PPI scanning mode degrades the space resolution by skipping certain elevation angles to reduce time required for scanning the region around the radar. These gaps should be filled using an interpolation scheme to give meaningful information of weather at a given altitude.

Earlier CAPPI algorithms utilize a 'nearest neighbor' approach whereby the available radar resolution cell nearest to the CAPPI surface is taken to lie on the surface itself. This introduces artifacts into the display, including sharp jumps or boundaries between annular segments of the display. This scheme determines the display parameter at the CAPPI surface by making a weighted interpolation of the data from adjacent

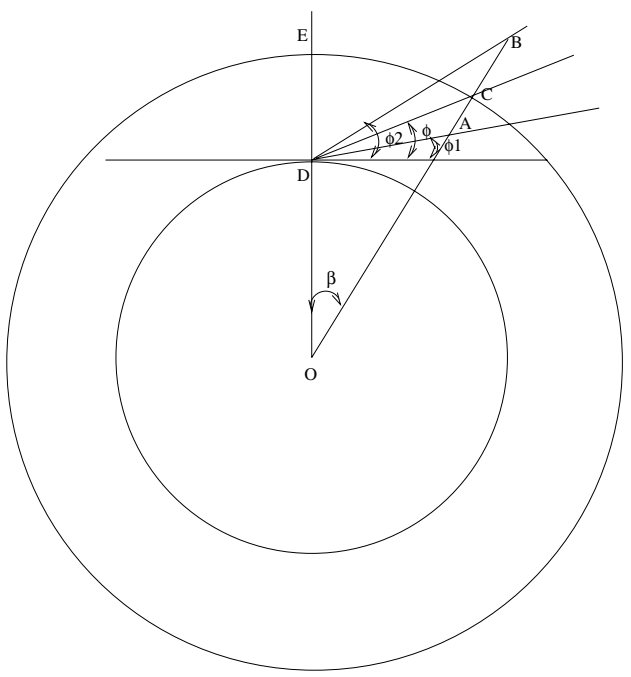

Fig. 2. Interpolation Scheme for CAPPI

scan elevations to obtain a smoother and more accurate CAPPI display.

In this paper, the CAPPI is constructed in a two-step process. The first step constructs the CAPPI scan line data along each azimuth angle of the radar, processing clockwise from 0 to $2 \pi$. In the second step the scan line data is presented to the PPI algorithm for coordinate scan conversion. Let $R_{e}$ be the radius of earth and $\mathrm{H}$ be the altitude above the earth surface at which CAPPI should be constructed. The scan line data along each azimuth angle is constructed by incrementing the distance from the north of radar $A B$ in equal step sizes, typically $1 \mathrm{~km}$ along the sphere of radius $R_{e}+\mathrm{H}$ as shown in $\operatorname{Fig}(1)$.

The CAPPI data should be constructed with reference to the geocentric coordinate system. Hence the coordinates should be converted from geocentric coordinate system to radar coordinate system for indexing the radar data. Let $\mathrm{O}$ denote the center of earth, $\overrightarrow{O A}$ denote direction of north of radar and $\beta$ denote the angle subtended by arc $\mathrm{AB}$ at center of the earth. Let $\mathrm{BC}$ be the perpendicular drawn from $\mathrm{B}$ to $\mathrm{OA}$ and $\mathrm{D}$ be the origin of the radar. The elevation angle $\phi$ of the radar is computed for each increment in $\mathrm{AB}$ using equation( 1). The arc length from north of radar $A B$ for any elevation angle $\phi$ is calculated using equation( 2 ).

$$
\begin{array}{r}
\beta=\frac{A B}{R_{e}+H} ; B C=\left(R_{e}+H\right) \sin (\beta) \\
C D=\left(R_{e}+H\right) \cos (\beta)-R_{e} ; \phi=\tan ^{-1}\left(\frac{C D}{B C}\right) \\
\beta=\cos ^{-1}\left(\frac{R_{e} \cos (\phi)}{R_{e}+H}\right)-\phi ; A B=\left(R_{e}+H\right) \times \beta
\end{array}
$$

However, elevation angle $\phi$ may have been skipped during the radar scan operation. Let $\phi_{1}$ and $\phi_{2}$ denote the elevation angles of the radar adjacent to angle $\phi$ as shown in Fig. 2. Let $\mathrm{O}$ and $\mathrm{D}$ denote the center of earth and radar respectively 
while $\overrightarrow{O E}$ denotes the direction of north of radar. The CAPPI data at angle $\phi$ can be calculated as the weighted average of radar resolution volume data from elevation angles $\phi_{1}$ and $\phi_{2}$. Let EC denote the arc length from the north of radar and $\beta$ denote the angle subtended by EC at O. Let OC intersect radar data at elevation angles $\phi_{1}$ and $\phi_{2}$ in A and B respectively. On applying sine rule in $\triangle \mathrm{BCD}$, the distance between $\mathrm{C}$ and $\mathrm{B}$ can be calculated using equation( 3). On applying sine rule in $\triangle \mathrm{ACD}$, the distance between $\mathrm{C}$ and $\mathrm{A}$ can be calculated using equation( 4). If $X$ and $Y$ denote the resolution volume data at $\mathrm{A}$ and $\mathrm{B}$ respectively, the CAPPI data $Z$ at $\mathrm{C}$ is computed using equation( 5 ).

$$
\begin{gathered}
B C=D C \times \frac{\sin \left(\phi_{2}-\phi\right)}{\sin \left(\frac{\pi}{2}-\left(\phi_{2}+\beta\right)\right)} \\
A C=D C \times \frac{\sin \left(\phi-\phi_{1}\right)}{\sin \left(\frac{\pi}{2}+\phi_{1}+\beta\right)} \\
Z=\frac{((B C \times X)+(A C \times Y))}{(B C+A C)}
\end{gathered}
$$

The CAPPI data for increments in $\mathrm{AB}$ between the north of radar and the highest elevation angle of radar is taken from the radar resolution volume data for the highest elevation angle. The CAPPI data for increments in $\mathrm{AB}$ between the lowest elevation angle of radar and the elevation angle $0^{\circ}$ is taken from the radar resolution volume data for the lowest elevation angle. The bounds on $\mathrm{AB}$ for all the elevation angles of radar at a given altitude are computed using equation( 2). These pre-computed bounds are used for determining the adjacent elevation angles for increments in $\mathrm{AB}$, the scheme avoids computation of these angles for each increment in $\mathrm{AB}$.

\section{LATITUde-Longitude CoORdinAte System}

A cartesian coordinate system is completely defined by the direction of three axes and the position of the origin. However, cartesian coordinate system would be very inconvenient to most users e.g. cartographers, surveyors, engineers, sea and land navigators. The primary disadvantage is that an increase of height $h$ from the surface of the earth does not convert to an increase in the value of $z$ only, except at the north pole. On the equator, the value of $z$ would remain unchanged irrespective of the value of $h$.

A latitude-longitude coordinate system offers a convenient grid for mosaicing data at various altitudes. The latitude $\lambda$ is the inclination of the normal that contains the point under consideration with respect to the equatorial plan. The longitude $\phi$ of a point is the angle between the meridian plan and the meridian of reference. The proposed scheme samples the CAPPI data using a latitude-longitude grid and uses it for constructing the mosaic.

Let $R_{e}$ denote the radius of the earth and $H$ be altitude above the surface of the earth at which the mosaic is to be constructed. Let $\left(\lambda_{i}, \phi_{i}\right)$ be the latitude-longitude location of the radar $i$. The coordinates $\left(\left(\lambda_{\text {min }_{i}}, \phi_{\text {min }_{i}}\right),\left(\lambda_{\text {max }_{i}}, \phi_{\text {max }_{i}}\right)\right)$ of the Minimum Bounding Rectangle (MBR) of radar coverage can be calculated as

$$
\begin{gathered}
\triangle \lambda=\cos ^{-1}\left(\frac{R_{e}}{R_{e}+H}\right) ; \Delta \phi_{i}=\sin ^{-1}\left(\frac{\sin (\triangle \lambda)}{\cos \left(\lambda_{i}\right)}\right) \\
\lambda_{\text {min }_{i}}=\lambda_{i}-\triangle \lambda ; \lambda_{\max _{i}}=\lambda_{i}+\triangle \lambda \\
\phi_{\min _{i}}=\phi_{i}-\triangle \phi_{i} ; \phi_{\max _{i}}=\phi_{i}+\triangle \phi_{i}
\end{gathered}
$$

The coordinates $\left(\left(\lambda_{\min }, \phi_{\min }\right),\left(\lambda_{\max }, \phi_{\max }\right)\right)$ of the MBR for all the $N$ radars can be calculated as

$$
\begin{aligned}
\lambda_{\min } & =\arg \min _{i=1,2, \ldots, N} \lambda_{\text {min }_{i}} \\
\lambda_{\text {max }} & =\arg \max _{i=1,2, \ldots, N} \lambda_{\text {max }_{i}} \\
\phi_{\text {min }} & =\arg \min _{i=1,2, \ldots, N} \phi_{\text {min }_{i}} \\
\phi_{\text {max }} & =\arg \max _{i=1,2, \ldots, N} \phi_{\max _{i}}
\end{aligned}
$$

\section{Mosaicing of Scalar Data}

The overlapping radars at a point should be determined dynamically. For scalar data, the average of the values for the overlapping radars at a point is calculated and is displayed at that point. The algorithm for constructing the mosaic of scalar data with data from $N$ radars is

1) Determine the MBR coordinates $\left(\left(\lambda_{\text {min }_{i}}, \phi_{\text {min }_{i}}\right)\right.$, $\left.\left(\lambda_{\max _{i}}, \phi_{\max _{i}}\right)\right)$ for each $\operatorname{radar} i(1 \leq i \leq N)$ using equation( 6).

2) Determine $\left(\left(\lambda_{\min }, \phi_{\min }\right),\left(\lambda_{\max }, \phi_{\max }\right)\right)$ using equation( 7).

3) For each $(\lambda, \phi)$ such that $\lambda_{\min } \leq \lambda \leq \lambda_{\max }$ and $\phi_{\min } \leq$ $\phi \leq \phi_{\max }$ repeat steps 4 to 7 .

4) Set sum of resolution volume data $s$ and number of overlapping radars $n$ to zero.

5) For each radar $i$ such that $1 \leq i \leq N$ repeat step 6 .

6) Increment $n$ by one and add the resolution volume data to $s$ if $\lambda_{\text {min }_{i}} \leq \lambda \leq \lambda_{\max _{i}}$ and $\phi_{\min _{i}} \leq \phi \leq \phi_{\max _{i}}$.

7) Divide $s$ by $n$ and assign it to the mosaic data at $(\lambda, \phi)$, if $n \neq 0$.

\section{Reconstruction and Mosaicing of Vector Data}

For vector data, the vector should be constructed from the values of overlapping radars. An ideal strategy would be extracting the wind velocity in two or three dimensions depending on the combination of radars for a given element in space. However, if a given element in space is visible from more than three radars, the over-determined system of equations should be solved to obtain three dimensions of wind velocity. The vector is usually represented by an arrow, with the arrow head pointing towards the direction of the vector. Hence, the vector cannot be displayed at every point and should be displayed for a region. This scheme displays the vector over a square block region.

The mosaic consisting of four overlapping radars I, II, III and IV is shown in Fig. 3 and the regions along with the overlapping radars are shown in Table I. The vector for the square block consisting of a single region can be determined easily. However, a square block may contain multiple regions as illustrated by the square block $B$ consisting of regions 7,8 , 


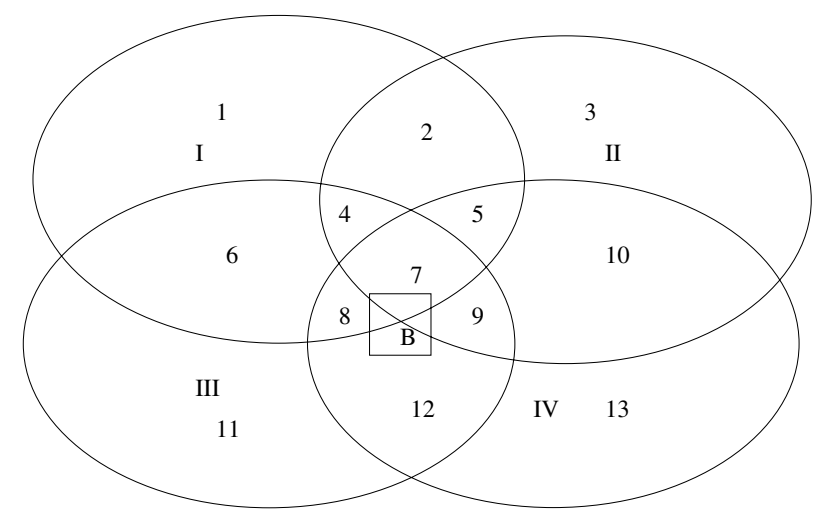

Fig. 3. Overlapping radar regions

9 and 12. This scheme determines the region with maximum area within that block and constructs the vector using the data from radars corresponding to that region. If the region with maximum area comprises of three radars (e.g. regions 4 , $5,8,9)$ or more than three radars (e.g. region 7 ), the three dimensional vector can be constructed. The two dimensional vector can be constructed for a region with two overlapping radars (e.g. regions 2, 6, 10, 12).

The position vector from the origin of the radar to the center of the block is calculated for all the radars corresponding to the maximum occurring region. The average of the values is calculated independently for all the radars in that region. The dot product of the position vector and wind vector gives the radial component of velocity. However, since the vector is displayed over a square region, the radial component is taken as the average of the values. These equations are solved to obtain the actual vector in two or three dimensions depending on the number of overlapping radars. The three dimensional vector is constructed using least squares solution when the number of overlapping radars is more than three. If the set of equations is written in matrix notation (equation( 8)), then the least squares solution is found by solving equation(9) where $A^{T}$ denotes the transpose of $\mathrm{A}$.

$$
\begin{aligned}
A X & =B \\
A^{T} A X & =A^{T} B
\end{aligned}
$$

Let $n \times n$ be the size of square block chosen for constructing vector data. The algorithm for extracting the wind vectors with data from $N$ radars is

1) Determine the MBR coordinates $\left(\left(\lambda_{\text {min }_{i}}, \phi_{\text {min }_{i}}\right)\right.$, $\left.\left(\lambda_{\max _{i}}, \phi_{\text {max }_{i}}\right)\right)$ for each $\operatorname{radar} i(1 \leq i \leq N)$ using equation( 6).

2) Determine $\left(\left(\lambda_{\min }, \phi_{\min }\right),\left(\lambda_{\max }, \phi_{\max }\right)\right)$ using equation( 7).

3) Divide the latitude and longitude grid between $\left(\left(\lambda_{\min }\right.\right.$, $\left.\left.\phi_{\min }\right),\left(\lambda_{\max }, \phi_{\max }\right)\right)$ into square blocks of size $\mathrm{n} \times \mathrm{n}$.

4) For each square block of size $n \times n$ repeat steps 5 to 8.

5) Determine the combination of $M$ overlapping radars that contribute to maximum region within that block.
TABLE I

OVERLAPPING RADAR REGIONS

\begin{tabular}{|c|l|}
\hline Region & Overlapping radars \\
\hline 1 & I \\
\hline 2 & I and II \\
\hline 3 & II \\
\hline 4 & I, II, and III \\
\hline 5 & I, II, and IV \\
\hline 6 & I and III \\
\hline 7 & I, II, III, and IV \\
\hline 8 & I, III, and IV \\
\hline 9 & II, III, and IV \\
\hline 10 & II and IV \\
\hline 11 & III \\
\hline 12 & III and IV \\
\hline 13 & IV \\
\hline
\end{tabular}

6) Calculate the average $A_{i}$ of the resolution cell values independently for each radar $i$ corresponding to the maximum region.

7) Let the wind velocity be $\mathbf{V}$. Calculate the dot product of $\mathbf{V}$ and unit position vector $\mathbf{P}_{i}$ from each radar $i$ corresponding to the maximum region and express it as

$$
\mathbf{V} \bullet \mathbf{P}_{i}=A_{i}
$$

8) In the case of two or three overlapping radars (i.e $M$ $=2,3$ ), solve the equations obtained in step 7 directly. However, if the number of overlapping radars is more than 3 (i.e. $M \geq 4$ ), solve them using Least Squares method to obtain the three components of the vector.

\section{CONCLUSION}

This paper presents a scheme for combining Doppler velocity data from multiple WSR-88D radars. A novel interpolative scheme for spherical-earth CAPPI format is presented. Several practical problems encountered in combining and mosaicing velocity data over large regions are addressed.

Results based on application of the methods to actual radar data are not included in the paper because of limitations of space and color graphics. Such results will be presented in the interactive session.

\section{REFERENCES}

[1] R. J. Doviak and D. S. Zrnic, Doppler Radar and Weather Observations, 2nd ed. San Diego, CA: Academic Press, 1993.

[2] P. R. Mahapatra, Aviation Weather Surveillance Systems: Advanced Radar and Surface Sensors for Flight Safety and Air Traffic Management. London, UK: IEE Press, 1999.

[3] J. Zhang, J. J. Gourley, K. Howard, and B. Maddox, "Three-dimensional gridding and mosaic of reflectivities from multiple wsr-88d radars," in Proc. 30th International Conference on Radar Meteorology, Munich, Germany, July 2001, pp. $719-721$.

[4] R. Watson and D. Bebbington, "Combining ground based meteorological radar data from multiple overlapping sites," in Proc. International Geoscience and Remote Sensing Symposium, vol. 3, Florence, Italy, July 1995, pp. 1660 - 1662.

[5] M. J. Logan, "Apparatus and method for constructing a mosaic of data," U.S. Patent 5742 297, April 21, 1998.

[6] J. P. Andrieu, D. Gault, and J. C. Henri, "Method and device for the display of several radar images in a single mosaic," U.S. Patent 5036326 , July 30, 1991. 